\title{
A Note on the Matching Polytope of a Graph
}

\author{
N.M.M. ABREU ${ }^{1}$, L.M.G.C. COSTA ${ }^{2}$, C.H.P. NASCIMENTO ${ }^{3}$ and L. PATUZZI ${ }^{4}$ \\ Received on April 9, 2018 / Accepted on December 7, 2018
}

\begin{abstract}
The matching polytope of a graph $G$, denoted by $\mathscr{M}(G)$, is the convex hull of the set of the incidence vectors of the matchings of $G$. The graph $\mathscr{G}(\mathscr{M}(G))$, whose vertices and edges are the vertices and edges of $\mathscr{M}(G)$, is the skeleton of the matching polytope of $G$. In this paper, for an arbitrary graph, we prove that the minimum degree of $\mathscr{G}(\mathscr{M}(G))$ is equal to the number of edges of $G$, generalizing a known result for trees. From this, we identify the vertices of the skeleton with the minimum degree and we prove that the union of stars and triangles characterizes regular skeletons of the matching polytopes of graphs.
\end{abstract}

Keywords: regular graph, matching polytope, degree of matching.

\section{INTRODUCTION}

Let $G=G(V, E)$ be a simple graph with vertex set $V=\left\{v_{1}, v_{2}, \ldots, v_{n}\right\}$ and set of edges $E=$ $\left\{e_{1}, e_{2}, \ldots, e_{m}\right\}$. For each $k, 1 \leq k \leq m, e_{k}=v_{i} v_{j}$ is an incident edge to the adjacent vertices $v_{i}$ and $v_{j}, 1 \leq i<j \leq n$. The set of adjacent vertices of $v_{i}$ is $N_{G}\left(v_{i}\right)$, called the neighborhood of $v_{i}$, whose cardinality $d\left(v_{i}\right)$ is the degree of $v_{i}$. A vertice $v_{i} \in V$ is said to be pendant if $d\left(v_{i}\right)=1$. An edge of $G$ is said to be pendant if one of its vertices has only one neighbor. For a given edge $e_{k}$, the set of adjacent edges of $e_{k}$ is denoted $I\left(e_{k}\right)$.

Two non adjacent edges are disjoint and a set of pairwise disjoint edges $M$ is a matching of $G$. An unitary edge set is a one-edge matching and the empty set is the empty matching, $\varnothing$. A vertex $v \in V$ is said to be $M$-saturated if there is an edge of $M$ incident to $v$. Otherwise, $v$ is said to be an $M$-unsaturated vertex. A perfect matching $M$ is one for which every vertex of $G$ is $M$-saturated.

For a natural number $k$, a path with length $k, P_{k+1}$ (or simply $P$ ), is a sequence of distinct vertices $v_{1} v_{2} \ldots v_{k} v_{k+1}$ such that, for $1 \leq i \leq k, e_{i}=v_{i} v_{i+1}$ is an edge of $G$. A cycle with length $k, C_{k}$ (or

\footnotetext{
*Corresponding author: Carlos Henrique Pereira do Nascimento - E-mail: carloshenrique@id.uff.br-https : //orcid. org/0000-0001-6023-2397

${ }^{1}$ Engenharia de Produção, COPPE, Universidade Federal do Rio de Janeiro, Rio de Janeiro, Brasil. E-mail: nairabreunovoa@gmail.com.

${ }^{2}$ Colégio Pedro II, Rio de Janeiro, Brasil. E-mail: lmgccosta@gmail.com.

${ }^{3}$ Departamento de Matemática, Instituto de Ciências Exatas, Universidade Federal Fluminense, Volta Redonda, Brasil.

E-mail: carloshenrique@id.uff.br.

${ }^{4}$ Instituto de Matemática, Universidade Federal do Rio de Janeiro, Rio de Janeiro, Brasil.
} 
simply $C$ ), is obtained from $P_{k}$ by adding the edge $v_{1} v_{k}$. If $k$ is odd, $C_{k}$ is said to be an odd cycle. Otherwise, $C_{k}$ is an even cycle. A path or a cycle can also be denoted by an ordered sequence of their respective edges. The ordering of the edges is given by the sequence of the vertices of the path or the cycle. Given a matching $M$ in $G$, a path $P$ is an $M$-alternating path in $G$ iff it contains, alternately, edges from $E \backslash M$ and $M$. A cycle $C$ is $M$-alternating iff $C$ is an even cycle and it contains alternately edges from $E \backslash M$ and $M$. For more basic definitions and notations of graphs, see [2], [5] and, for matchings, see [8].

A polytope of $\mathbb{R}^{n}$ is the convex hull $\mathscr{P}=$ convex $\left\{x_{1}, \ldots, x_{r}\right\}$ of a finite set of vectors $x_{1}, \ldots, x_{r} \in$ $\mathbb{R}^{n}$. Given a polytope $\mathscr{P}$, the skeleton of $\mathscr{P}$ is a graph $\mathscr{G}(\mathscr{P})$ whose vertices and edges are, respectively, vertices (faces of dimension 0 ) and edges (faces of dimension 1 ) of $\mathscr{P}$.

For the ordered set $E$ of $m$ edges of a graph, $\mathbb{R}^{E}$ is the vector space of real-valued vectors indexed by the elements of $E$ whose dimension is $\operatorname{dim}\left(\mathbb{R}^{E}\right)=m$. For $F \subset E$, the incidence vector of $F$ is defined as follows:

$$
\chi_{F}(e)= \begin{cases}1, & \text { if } e \in F \\ 0, & \text { otherwise }\end{cases}
$$

In general, we identify each subset of edges with its respective incidence vector. The matching polytope of $G, \mathscr{M}(G)$, is the convex hull of the incidence vectors of the matchings in $G$. For more definitions and notations of polytopes, see [7].

Two matchings $M$ and $N$ are said adjacent, $M \sim N$, if and only if their correspondent vertices $\chi_{M} \equiv M$ and $\chi_{N} \equiv N$ are adjacent in the skeleton of the matching polytope. The degree of a matching $M$, denoted $d(M)$, is the degree of the correspondent vertex in $\mathscr{G}(\mathscr{M}(G))$.

Given two sets $A$ and $B$, the symmetric difference $A \Delta B$ is defined by $(A \cup B) \backslash(A \cap B)$. Next theorems characterize the adjacency of two matchings $M$ and $N$ by their symmetric difference $M \Delta N$.

Theorem 1. ([3]) Two distinct matchings $M$ and $N$ of a graph $G$ are adjacent in the matching polytope $\mathscr{M}(G)$ if and only if $M \Delta N$ is a connected subgraph of $G$.

Theorem 2. ([9]) Two distinct matchings $M$ and $N$ of a graph $G$ are adjacent in the matching polytope $\mathscr{M}(G)$ if and only if $M \Delta N$ is a path or a cycle (even cycle) in $G$.

Figure 1 displays the cycle $C_{4}$ and the skeleton of its matching polytope, $\mathscr{G}\left(\mathscr{M}\left(C_{4}\right)\right)$. Since $M_{1} \Delta M_{2}$ is a path and $M_{5} \Delta M_{6}$ is the cycle $C_{4}, M_{1} \sim M_{2}$ and $M_{5} \sim M_{6}$ in $\mathscr{G}\left(\mathscr{M}\left(C_{4}\right)\right)$. However, $M_{1} \nsim M_{3}$, once $M_{1} \Delta M_{3}$ is a disconnected subgraph of the cycle.

Let $T$ be a tree with $n$ vertices. The acyclic Birkhoff polytope $\Omega_{n}(T)$ is the set of $n \times n$ doubly stochastic matrices $A=\left[a_{i j}\right]$ such that the diagonal entries of $A$ correspond to the vertices of $T$ and each positive entry of $A$ is either on the diagonal or on a position corresponding to an edge of $T$. The matching polytope $\mathscr{M}(T)$ and the acyclic Birkhoff polytope $\Omega_{n}(T)$ are affinely isomorphic 

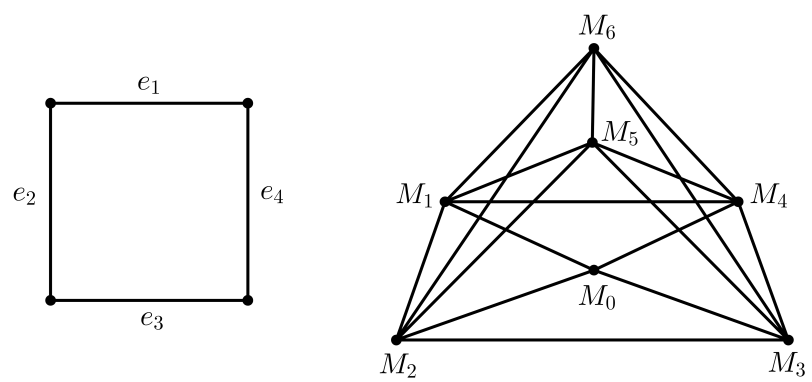

$$
\begin{aligned}
& M_{0}=\emptyset \quad M_{1}=\left\{e_{1}\right\} \quad M_{2}=\left\{e_{2}\right\} \quad M_{3}=\left\{e_{3}\right\} \\
& M_{4}=\left\{e_{4}\right\} \quad M_{5}=\left\{e_{1}, e_{3}\right\} \quad M_{6}=\left\{e_{2}, e_{4}\right\}
\end{aligned}
$$

Figure 1: $C_{4}$ and $\mathscr{G}\left(\mathscr{M}\left(C_{4}\right)\right)$

[4]. The skeleton of $\Omega_{n}(T)$ was studied in [1] and [6] and, in the sequence, we highlight the following contribution given by them.

Theorem 3. ([1]) If $T$ is a tree with $n$ vertices, the minimum degree of $\mathscr{G}(\mathscr{M}(T))$ is $n-1$. Moreover, for a matching $M$ of $T, d(M)=n-1$ if and only if $M=\varnothing$ or every edge of $M$ is a pendant edge of $T$.

In this paper, we generalize the above result for $G$, where $G$ is an arbitrary graph. Based on it, we prove two theorems of characterization: the first identifies the matchings of $G$ with the minimum degree and the second gives a necessary and sufficient condition about $G$ in order to have $\mathscr{G}(\mathscr{M}(G))$ as a regular graph.

\section{THE DEGREE OF A MATCHING}

In the present section we generalize the results from Abreu et al. [1]. From Theorem $2, d(\varnothing)=m$ in $\mathscr{G}(\mathscr{M}(G))$. In fact, there are $m$ one-edge matchings, thus $\varnothing$ as exactly $m$ neighbors. On the other hand, let $M \neq \varnothing$ be a matching of $G$ and $e$ be an edge of $G$. If $e$ is an edge of $M$ then $M$ is adjacent to the matching $N=M \backslash\{e\}$. If $e$ is not an edge of $M$ then $M$ is adjacent to the matching $K=(M \backslash\{f: f$ is an edge in $M$ adjacent to $e\}) \cup\{e\}$. Therefore, the degree of $M$ is greater than or equal to the number of edges.

Let $G$ be a graph with a matching $M$ and $P$ be an $M$-alternating path with at least two vertices. We say that:

(i) $P$ is an $o o-M$-path if its pendant edges belong to $M$;

(ii) $P$ is a $c c-M$-path if its pendant vertices are both $M$-unsaturated;

(iii) $P$ is an $o c-M$-path if one of its pendant edges belongs to $M$ and one of its pendant vertex is $M$-unsaturated. 
An $M$-alternating path $P$ is called an $M$-good path when $P$ is one of the paths defined above. Moreover, if $C$ is a cycle of $G$, then $C$ is said to be an $M$-good cycle when $C$ is an $M$-alternating cycle. In this case, $M \cap E(C)$ is a perfect matching of $C$.

In Figure $2, M=\left\{e_{1}, e_{3}, e_{6}\right\}$ is a perfect matching of the graph. Because $M$ is a perfect matching, there is no unsaturated vertex. Therefore, there is no $c c-M$-good path nor $o c-M$-good path in $G$. However, $e_{1} e_{2} e_{3}$ is an oo-M-good path of $G$ but $e_{1} e_{2}$ is not an $M$-good path. Moreover, $e_{1} e_{2} e_{3} e_{4}$ is an $M$-good cycle.

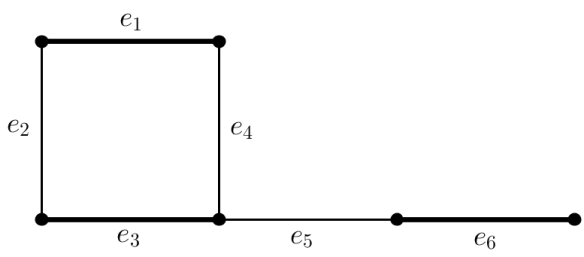

Figure 2: The matching $M=\left\{e_{1}, e_{3}, e_{6}\right\}$ and the $M$-good cycle $C=e_{1} e_{2} e_{3} e_{4}$.

If the symmetric difference $M \Delta N$ is a path, where $M$ and $N$ are any two matchings, necessarily it is an $M$-good path. Note also that when $M \Delta N$ is a cycle then it is $M$-good and for $N_{1} \neq N_{2}$ we have $M \Delta N_{1} \neq M \Delta N_{2}$. Based on this, the next theorem is a direct consequence from Theorems 1 and 2.

Theorem 4. Let $M$ be a matching of a graph $G$. The degree of $M$ in the skeleton $\mathscr{G}(\mathscr{M}(G))$ is given by the sum of the number of paths and cycles M-good.

A matching $M$ is said to be a strict matching when two edges of $M$ have no a common incident edge, i.e.,

$$
\forall e, f \in M: e \neq f \Longrightarrow I(e) \cap I(f)=\varnothing
$$

In the next theorem, we give a formulae to compute the degree of a strict matching $M$ that depends only on degree and neighbors of the vertices $M$-saturated. Before, we observe that if $M$ is a strict matching and $C$ is a cycle of $G$ then $C$ is a not $M$-alternating cycle. Also note that if $P$ is an $M$-good path then $P$ has at most one edge in $M$ and its length is at most 3.

Proposition 5. Let $M=\left\{e_{1}, \ldots, e_{s}\right\}$ be a strict matching of a graph $G=(V, E)$ and let $e_{i}=u_{i} v_{i}$ where $u_{i}, v_{i} \in V$ for each $1 \leq i \leq s$. The degree of $M$ is

$$
d(M)=k+\sum_{i=1}^{s}\left(d\left(u_{i}\right) d\left(v_{i}\right)-\left|N\left(u_{i}\right) \cap N\left(v_{i}\right)\right|\right),
$$

where $k \geq 0$ is the number of edges that have no vertex in common with any edge of $M$.

Proof. Let $M$ be as in the statement of Proposition 5. In this case, $G$ does not have $M$-good cycles and, if $P$ is an $M$-good path, $P$ has at most length 3 with at most one edge of $M$. Hence, if $P$ is an $M$-good path of $G, P$ has to satisfy one of the cases below. 
(1) $P$ is an $o o$-M-path. Then, $P=e$ such that $e \in M$. There are $s$ of these paths in $G$;

(2) $P$ is an $o c$-M-path. So, $P=e f$ such that $e \in M$ and $f \notin M$. Of course, for some $i, 1 \leq i \leq s$, we have $e=u_{i} v_{i}$ and $f$ is incident to $u_{i}$ or to $v_{i}$. There are $\left(d\left(u_{i}\right)-1\right)+\left(d\left(v_{i}\right)-1\right)$ of these paths;

(3) If $P$ is a $c c$-M-path, we have to consider two possibilities for $P$. Firstly, $P=f e g$ with $e \in M$ and $f, g \notin M$. So, for some $i, 1 \leq i \leq s, e=u_{i} v_{i}$ such that $f$ is incident to $u_{i}$ and $g$ is incident to $v_{i}$. In this case, there are $\left(d\left(u_{i}\right)-1\right) \cdot\left(d\left(v_{i}\right)-1\right)-\left|N\left(u_{i}\right) \cap N\left(v_{i}\right)\right|$ of such paths. The second possibility is $P=f$ with $f \notin M$. Since $P$ is a $c c$-M-path, for each $e \in E$ that is incident to $f, e \notin M$. The number of such edges is equal to the number $k$ defined in the statement of Proposition 5.

From the itens (1), (2) and (3), we obtain

$$
\begin{gathered}
d(M)=s+k+\sum_{i=1}^{s}\left(\left(d\left(u_{i}\right)-1\right)+\left(d\left(v_{i}\right)-1\right)+\left(d\left(u_{i}\right)-1\right)\left(d\left(v_{i}\right)-1\right)-\left|N\left(u_{i}\right) \cap N\left(v_{i}\right)\right|\right)= \\
=k+\sum_{i=1}^{s}\left(d\left(u_{i}\right) d\left(v_{i}\right)-\left|N\left(u_{i}\right) \cap N\left(v_{i}\right)\right|\right) .
\end{gathered}
$$

Theorem 6. Let $M$ and $N$ be matchings of a graph $G$. If $M \subset N$, then $d(M) \leq d(N)$.

Proof. Let $M$ and $N$ be matchings of a graph $G$ such that $N=M \cup\{e\}$, where $e \in E$. Consider $\mathbb{B}_{M}$ the sets of $M$-good paths and $M$-good cycles of $G$. Similarly, define $\mathbb{B}_{N}$. From Theorem 4 , $d(M)=\left|\mathbb{B}_{M}\right|$ and $d(N)=\left|\mathbb{B}_{M}\right|$. Build the function $\varphi_{e}: \mathbb{B}_{M} \rightarrow \mathbb{B}_{N}$ such that, for every cycle $C \in \mathbb{B}_{M}, \varphi_{e}(C)=C$ and, for every path $P \in \mathbb{B}_{M}$,

$$
\varphi_{e}(P)= \begin{cases}P, & \text { if } V(e) \cap V(P)=\varnothing ; \\ P \cup\{e\}, & \text { if } V(e) \cap V(P)=u, \text { where } u \text { is one of the endpoints of } P .\end{cases}
$$

In fact, since $P$ is an $M$-good path, $M \cup\{e\}$ is a matching and $e \notin M$, then there are only two possible cases: either $V(e) \cap V(P)=\varnothing$ or $V(e) \cap V(P)=u$ (and therefore is $M$ unsaturated). By construction, for distinct paths or cycles belonging to $\mathbb{B}_{M}$, we have distinct images in $\mathbb{B}_{N}$. Then, $\varphi_{e}$ is an injective function and so, $d(M) \leq d(N)$.

In the general case, let $N \backslash M=\left\{e_{1}, e_{2}, \ldots, e_{k}\right\}, N_{1}=M \cup\left\{e_{1}\right\}, N_{2}=M \cup\left\{e_{1}, e_{2}\right\}, \ldots$, and $N_{k}=N$. By the same argument used before, we get $d(M) \leq d\left(N_{1}\right) \leq d\left(N_{2}\right) \leq \cdots \leq d\left(N_{k-1}\right) \leq d(N)$.

From the previous theorem applied to $M=\varnothing$, we can also obtain that if $G$ is a graph with $\mathrm{m}$ edges then the minimum degree of $\mathscr{G}(\mathscr{M}(G))$ is equal to $m$. 


\section{REGULAR MATCHINGS POLYTOPES}

In this section we characterize the graphs for which the skeletons are regular and all matchings of a graph with minimum degree.

An edge $e=u v$ of a graph $G$ is called a bind if $d(u)=d(v)=2$ and $|N(u) \cap N(v)|=1$. Note that if $e$ is a bind of $G, e$ is an edge of a triangle of graph. However, the reciprocal is not necessarily true.

Proposition 7. Let $G$ be a graph with $m$ edges and $M=\{e\}$ be a one-edge matching of $G$. The degree of $M$ is $d(M)=m$ if and only if $e$ is either a bind or a pendent edge of $G$.

Proof. Let $G$ be a graph with $m$ edges and $e=u v$ an edge of $G$. We know that $m-d(u)-d(v)+1$ is the number of edges that neither is incident to $u$ nor to $v$. From Proposition $5, d(\{e\})=$ $m-d(u)-d(v)+1+d(u) d(v)-t$, where $t=|N(u) \cap N(v)|$. Therefore, $d(\{e\})=m$ if and only if $d(u) d(v)-t=d(u)+d(v)-1$, i.e., $(d(u)-1)(d(v)-1)=t$. Since $d(u)>t$ and $d(v)>t$, $(d(u)-1)(d(v)-1) \geq t^{2}$. So, $(d(u)-1)(d(v)-1)=t$ if and only if $t=0$ or $t=1$. In the first case, $e$ is a pendant edge and, otherwise, $d(u)=d(v)=2$ and so, $e$ is a bind.

Note that, if $G$ is a graph without binds and pendant edges, the unique vertex of $\mathscr{G}(\mathscr{M}(G))$ with the minimum degree is $M=\varnothing$. It is not difficult to see that, except $K_{3}$, all 2-connected graphs satisfy this property.

Proposition 8. Let $G$ be a graph with $m$ edges and $M \neq \varnothing$ be a matching of $G$. Then, $d(M)=m$ if and only if $M$ has only pendant edges or binds.

Proof. Suppose there is $e \in M$ such that $e$ is neither a bind nor a pendant edge of $G$. So, from Proposition 7 and Theorem $6, m<d(\{e\}) \leq d(M)$. Consequently, $m \neq d(M)$. By the contrapositive, if $d(M)=m$, every edge of $M$ is a pendant edge or a bind of the graph.

Suppose now that $M$ is a matching of $G$ with $s$ edges such that if $e \in M, e$ is a bind or $e$ is a pendant edge of $G$. Let $N$ be a matching such that $N \sim M$ in $\mathscr{G}(\mathscr{M}(G))$. From here and by Theorem 2, $M \Delta N$ is an $M$-good path $P$ or an $M$-good cycle $C$ of $G$. Since $C$ is an even cycle, $C \neq K_{3}$. So, $C$ does not have binds. Consequently, $M \Delta N$ is a path. Concerning the path $P$, only its pendant edges can belong to $M$. Moreover, once $P$ is an alternated path, it has length at most length 3 . Hence, there are only the following possibilities to $P$ :

1. If $P$ is an $o o$-M-path, then $P=e$, where $e \in M$, or $P=e_{1} f e_{2}$, where $f \notin M$ and $I(f) \cap M=$ $\left\{e_{1}, e_{2}\right\}$. In the first case, there are $s$ possibilities to $P$ and, in the second, there are $t_{1}$ possibilities to $P$, where $t_{1}$ is the number of edges of $E \backslash M$ such that both terminal vertices are incident to an edge of $M$;

2. If $P$ is a $c c$-M-path, $P=f$, where $f \notin M$ and $I(f) \cap M=\varnothing$. Here, there are $t_{2}$ possibilities to $P$, where $t_{2}$ is the number of edges of $E \backslash M$ for which any edge of $M$ does not incident to the end vertices of those edges; 
3. Finally, if $P$ is an $o c$-M-path, then $P=e f$, where $f \notin M$ and $I(f) \cap M=\{e\}$. In this last case, there are $t_{3}$ possibilities to $P$, where $t_{3}$ is the number of edges of $E \backslash M$ which only one end vertex of edge is incident to some edge of $M$.

From (1), (2) and (3) possibilities above, $s+\sum_{i=1}^{3} t_{i}=s+|E \backslash M|=m$ is the number of the possibilities to have $P$ as an M-good path of $G$. From Theorem 4 it follows that $d(M)=m$.

Finally, next theorem proves that the union of stars and triangles characterizes regular skeletons of the matching polytopes of graphs.

Theorem 9. Let $G$ be a graph with $m$ edges. The skeleton $\mathscr{G}(\mathscr{M}(G))$ is an m-regular graph if and only if $G$ is a disjoint union of stars and triangles.

Proof. Suppose that $\mathscr{G}(\mathscr{M}(G))$ is an $m$-regular graph. Then, for every $e \in E$, we have $d(\{e\})=$ $d(\varnothing)=m$. Besides, by Proposition 7, this occurs only if $e$ is a bind or a pendant edge of $G$. So, $G$ be a graph that is a disjoint union of stars and triangles.

Suppose now that $G$ is a disjoint union of stars and triangles. Therefore, any matching of $G$ has only pendant edges or binds. From Proposition $8, \mathscr{G}(\mathscr{M}(G))$ is an $m$-regular graph.

Figure 3 displays the graph $G=K_{3} \cup S_{1,1}$ and its skeleton, $\mathscr{G}\left(\mathscr{M}\left(K_{3} \cup S_{1,1}\right)\right)$.
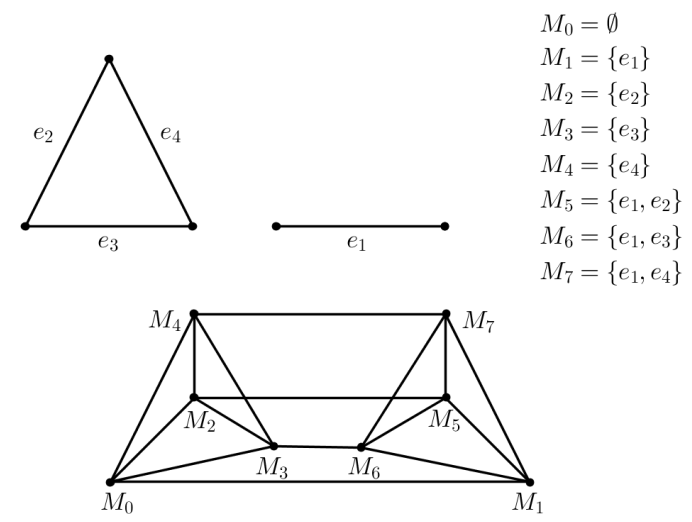

Figure 3: $G=K_{3} \cup S_{1,1}$ and $\mathscr{G}(\mathscr{M}(G))$

\section{FINAL CONSIDERATIONS}

In this paper we give a closed formula to compute the degree of a strict matching, i.e., a matching $M=\left\{e_{1}, \ldots, e_{k}\right\}$ such that $\forall i, j \in\{1, \ldots, k\}, e_{i}, e_{j} \in M, e_{i} \neq e_{j} \Rightarrow I\left(e_{i}\right) \cap I\left(e_{j}\right)=\varnothing$. Abreu 
et al. [1] give the minimum degree of the matching polytope for a tree. We generalize this result for any graph. We characterize all the graphs $G$ for which $\mathscr{G}(\mathscr{M}(G))$ is regular. Besides, in the last graph, we find all vertices with minimum degree. Finally, we emphasize that the problem of determination of the maximum degree of $\mathscr{G}(\mathscr{M}(G))$ is still unresolved.

\title{
5 ACKNOWLEDGMENT
}

The authors are grateful to anonymous referees for their suggestions and comments that improved substantially the quality of this article. This work was partially supported by CNPq, PQ-304177/2013-0 and Universal Project-442241/2014-3, and by the Portuguese Foundation for Science and Technology (FCT), CIDMA-project UID/MAT/04106/2013.

\begin{abstract}
RESUMO. O politopo de emparelhamentos de um grafo $G$, denotado por $\mathscr{M}(G)$, é o fecho convexo do conjunto dos vetores de incidência dos emparelhamentos de $G$. O grafo $\mathscr{G}(\mathscr{M}(G))$, cujos vértices e arestas são os vértices e arestas de $\mathscr{M}(G)$, é o esqueleto do politopo de emparelhamentos de $G$. Neste artigo, para um grafo arbitrário, nós provamos que o grau mínimo de $\mathscr{G}(\mathscr{M}(G))$ é igual ao número de arestas de $G$, generalizando um conhecido resultado para árvores. Além disso, nós identificamos os vértices do esqueleto que possuem grau mínimo e provamos que a união de estrelas e triângulos caracteriza esqueletos regulares de politopos de emparelhamentos de grafos.
\end{abstract}

Palavras-chave: grafo regular, politopo de emparelhamentos, grau de um emparelhamento.

\section{REFERENCES}

[1] N. Abreu, L. Costa, G. Dahl \& E. Martins. The skeleton of acyclic Birkhoff polytopes. Linear Algebra Appl., 457 (2014), 29-48.

[2] B. Bollobas. "Modern Graph Theory". Graduate Texts in Mathematics. Springer, New York (1998).

[3] V. Chvatal. On certain polytopes associated with graphs. Journal of Combinatorial Theory, B 18 (1975), $138-154$.

[4] L. Costa, C.M. da Fonseca \& E.A. Martins. The diameter of the acyclic Birkhoff polytope. Linear Algebra Appl, 428 (2008), 1524-1537.

[5] R. Diestel. "Graph Theory”. Springer-Verlag, New York (2000).

[6] R. Fernandes. Computing the degree of a vertex in the skeleton of acyclic Birkhoff polytopes. Linear Algebra Appl, 475 (2015), 119-133.

[7] B. Grunbaum. “Convex Polytopes”. Springer-Verlag, New York (2003).

[8] L. Lovasz \& M.D. Plumer. "Matching Theory". Ann. Discrete Math. 29 121. North-Holland, Amsterdam (1986).

[9] A. Schrijver. "Combinatorial optimization: polyhedra and efficiency". Algorithms and Combinatorics 24. Springer-Verlag, New York (2003). 